BASIC RESEARCH

\title{
The forces generated within the musculature of the left ventricular wall
}

\author{
P P Lunkenheimer, K Redmann, J Florek, U Fassnacht, C W Cryer, F Wübbeling, P Niederer, \\ R H Anderson
}

See end of article for authors' affiliations .....................

Correspondence to: Prof Dr med P P Lunkenheimer, Klinik und Poliklinik für ThoraxHerz- und Gefäßchirurgie, Domagkstraße 11, 48129 Münster, Munster, Germany; redmann@ uni-muenster.de

Accepted 19 May 2003

\begin{abstract}
Objectives: To test the hypothesis that two populations of myocardial fibres - fibres aligned parallel to the surfaces of the wall and an additional population of fibres that extend obliquely through the wall-when working in concert produce a dualistic, self stabilising arrangement.

Methods: Assessment of tensile forces in the walls of seven porcine hearts by using needle probes. Ventricular diameter was measured with microsonometry and the intracavitary pressure through a fluid filled catheter. Positive inotropism was induced by dopamine, and negative inotropism by thiopental. The preload was raised by volume load and lowered by withdrawal of blood. Afterload was increased by inflation of a balloon in the aortic root. The anatomical orientation of the fibres was established subsequently in histological sections.

Results: The forces in the fibres parallel to the surface decreased $20-35 \%$ during systolic shrinkage of the ventricle, during negative inotropism, and during ventricular unloading. They increased $10-30 \%$ on positive inotropic stimulation and with augmentation in preload and afterload. The forces in the oblique transmural fibres increased $8-65 \%$ during systole, on positive inotropic medication, with an increase in afterload and during ventricular shrinkage, and decreased $36 \%$ on negative inotropic medication. There was a delay of up to $147 \mathrm{~ms}$ in the drop in activity during relaxation in the oblique transmural fibres. Conclusion: Although the two populations of myocardial fibres are densely interwoven, it is possible to distinguish their functions with force probes. The delayed drop in force during relaxation in obliquely oriented fibres indicates that they are hindered in their shortening to an extent that parallels any increase in mural thickness. The transmural fibres, therefore, contribute to stiffening of the ventricular wall and hence to confining ventricular compliance.
\end{abstract}

A force probe can be serially positioned in skeletal muscle to assess the actively developed forces, by virtue of the discrete origin and insertion of the fibres. To date, however, it has not been thought appropriate to use such probes to assess the generation of forces within the myocardium. This is because, in contrast to skeletal muscle, the myocardium is a syncytium, with the fibres having no ordered origin or insertion. Instead, the myocardial mass is a densely branching three dimensionally netted contractile system. This basic structure, at first sight, seems to be inaccessible to any direct measurement of contractile forces. On this basis, taking as their precedent the classical rigorous estimation of Huisman, ${ }^{1}$ previous investigators have repudiated the possibility of coupling a mechanical measuring system to the myocardial tissues. We now question these assertions, just as we hesitate to accept the totality of Frank's cardiodynamic concept ${ }^{2}$ suggesting that intramural dynamic variables are comprehensively mirrored by haemodynamic data, such as intracavitary pressure and stroke volume. Instead, we hypothesised that, by using needle force probes similar to those that we earlier used in skeletal muscle, ${ }^{3}$ it is possible to investigate local contractile activity within the myocardium. We then sought to validate our hypothesis with histological evidence of the coupling of the needle probes to the myocardium.

Some researchers have tried to measure myocardial forces in the immediately subepicardial layers, where the main alignment of the fibres can be seen to be parallel to the surface. $^{45}$ The conclusions drawn from these studies, however, ignore the potential coupling of these fibres to a second population of myocardial fibres, which run obliquely, ${ }^{6-8}$ extending transmurally from the epicardium towards the endocardium. It is inappropriate to exclude these fibres when interpreting the global orientation of the ventricular myocardium and the force it generates.

Our hypothesis, therefore, is based on our belief that our probes, sensitive to lateral compression, would distinguish focally the alignment of the myocardial fibres. ${ }^{3}{ }^{10}$ We accept that the myocardial fibres, rather than functioning as finite elements, act as a highly cross linked syncytium. Despite this, the fibres exhibit a distinct major orientation, such that a well defined relation exists between the topography of the coupling and the measurable tensile force $\left(\mathrm{F}_{\mathrm{T}}\right) .^{11-14}$ We present evidence to show that the populations of fibres, although oriented in diverging directions, are activated synchronously, but not necessarily unanimously, in the direction of ventricular emptying. Together, they function in dualistic fashion to preserve ventricular shape, the fibres oriented in different directions putting each other under mutual stress in a fashion that can be compared to the driving myomechanics of the tongue.

\section{METHODS}

In seven female pigs with a mean (SD) weight of 90 (6) kg, under deep ketamine-barbital-halothane anaesthesia, a lateral thoracotomy was performed through the fifth left intercostal space. Myocardial mesh tension was assessed with needle probes $^{3} 9^{10}$ implanted almost parallel yet slightly inclined with respect to the epicardium into several levels of the superior, lateral, and inferior walls of the left ventricle. During the insertion of the $2.5 \mathrm{~mm}$ thick probe, a shield covers the lateral window. This shield is pulled back when 


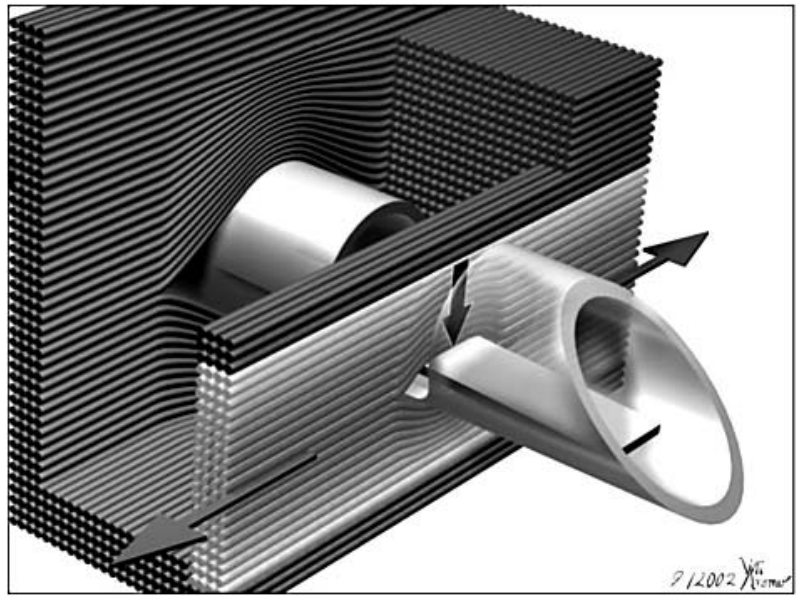

Figure 1 Illustration showing the $2.5 \mathrm{~mm}$ thick needle force probe inserted between the myocardial fibres. Also shown is the main direction of the development of contractile force along the axial alignment of the myocardial fibres, along with the perpendicular force vector that acts on the flexible bar within the lateral window, measured by strain gauges mounted on the flexible bar. The fibres are spread during implantation of the probe. Some fibres slip into the lateral window, loading there on the flexible bar. Only those (bright) fibres that are curved around the flexible bar contribute to the signal measured, the innermost curved fibres more than the outer, less curved fibres. In the background are shown the myocardial fibres wrapping around the trunk of the needle probe. Spreading of the tissue is more important in this area than in the indentation of the window.

the probe is in place. Some fibres then act on the flexible bar in the lateral window of the probe, providing a periodic force signal (fig 1). Since there is no membrane covering the window, the probe is insensitive to interstitial fluid pressure. The force is measured in milli-Newtons $(\mathrm{mN})$.

Before insertion, each force probe is tested to establish the stability of the signal, and to exclude any major "drift" in temperature, in saline warmed over a period of 30 minutes from $35-39^{\circ} \mathrm{C}$. During calibration of the probe, linearity is tested with a piston device, which itself is calibrated in the range of $0-100 \mathrm{mN}$. The device is fitted to the probe such that a fine piston exerts forces of 20,40,60,80, and $100 \mathrm{mN}$ on the inner bar of the probe through the centre of its lateral window. The resulting displacement of the bar by the piston in micrometres is indicated simultaneously.

The recorded value denotes $\mathrm{F}_{\mathrm{T}}$. As the probe is inserted, the netting of the fibres in the ventricular wall is slightly spread and hence deformed. Consequently, the signal has an initial fixed component caused by the insertion of the probe into the myocardium. This value serves as the diastolic control value from the beginning of the experiment. The second, variable, component of the signal then measures the variations in fibre tension generated during the ventricular cycle.

$\mathrm{F}_{\mathrm{T}}$ was evaluated as systolic minus diastolic value, velocity of early systolic rise $\left(\mathrm{dF}_{\mathrm{T}} / \mathrm{dt}_{\max }\right)$ and drop during relaxation $\left(\mathrm{dF}_{\mathrm{T}} / \mathrm{dt}_{\min }\right)$, and systolic intervals relative to the peak of the $\mathrm{R}$ wave in the surface ECG. In view of the difficulty in determining the exact point of initiation of mechanical activity, we did not attempt to determine the initial interval from the $\mathrm{R}$ wave to the onset of activity. We took representative measurements characteristic of two types of force behaviour. These were, firstly, an unloading type of signal that rose fast initially, leading to an early systolic maximum. After taking a sharp bend, the signal dropped to a second bend. From there it dropped to its diastolic level. A second auxotonic type of signal was also seen to have two bends, with the force rising between the bends, so that the force reaches its late systolic maximum at the second bend
Left ventricular pressure

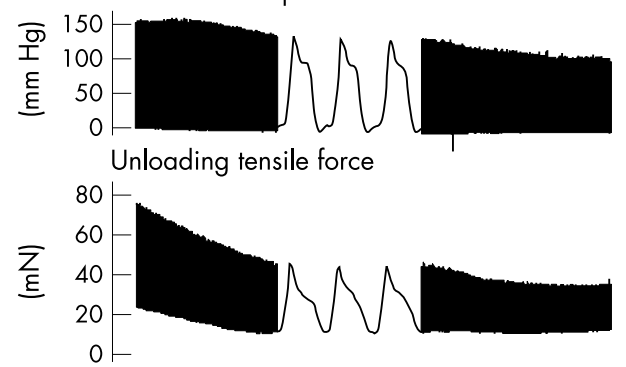

Auxotonic tensile force

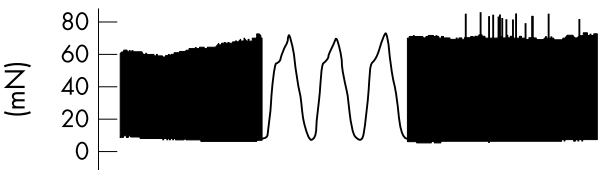

Left ventricular diameter

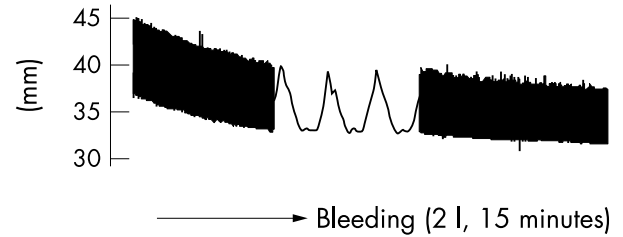

Figure 2 Continuous recording of left ventricular pressure, one force of the unloading type and one auxotonic force in the left ventricular wall, and left ventricular outer diameter during the withdrawal of 2 litres of blood within 15 minutes. Note the typical shapes of the unloading and auxotonic types of tensile forces. Note also that the auxotonic force increases while the ventricular diameter decreases.

(fig 2). Our histological investigations (see below) showed that the auxotonic pattern is associated with coupling of the probe to the oblique transmural fibres of the myocardial network (fig 3). We succeeded in evaluating one auxotonic $F_{T}$ in all animals, at least during massive changes in blood volume (figs 4 and 5).
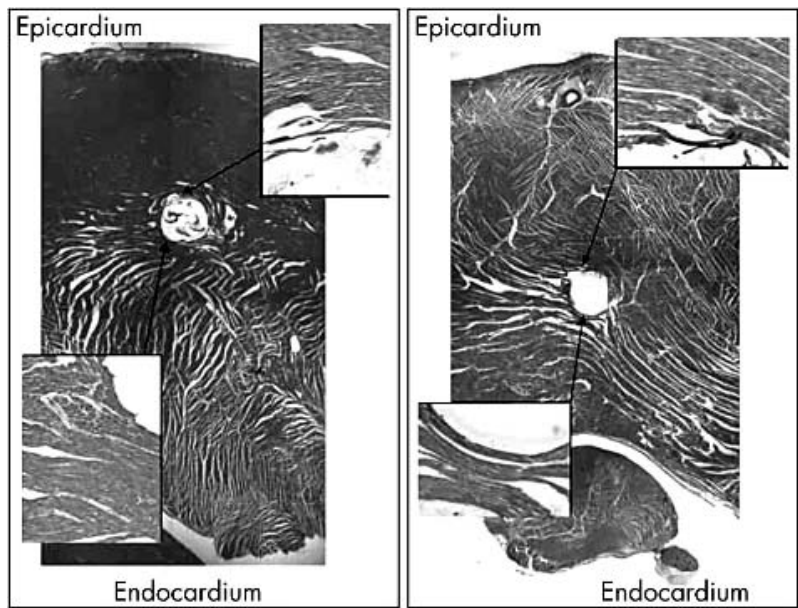

Figure 3 Cross sections through two sites of implantation of force probes into the left ventricular wall. The left panel shows the alignment of the fibres running parallel to the epicardium. In this area, the measured signal was of the unloading type. The right panel, in contrast, shows fibres that take a strongly oblique and transmural course. A signal of the auxotonic type was measured at this site. Note that the zone of implantation in the right section from near the left ventricular base is situated beyond the mid-layers, whereas in the left section it is situated at the limits of the outer to the mid-third of the wall. The incidence of auxotonic signals is higher in the deeper than in the superficial layers. 

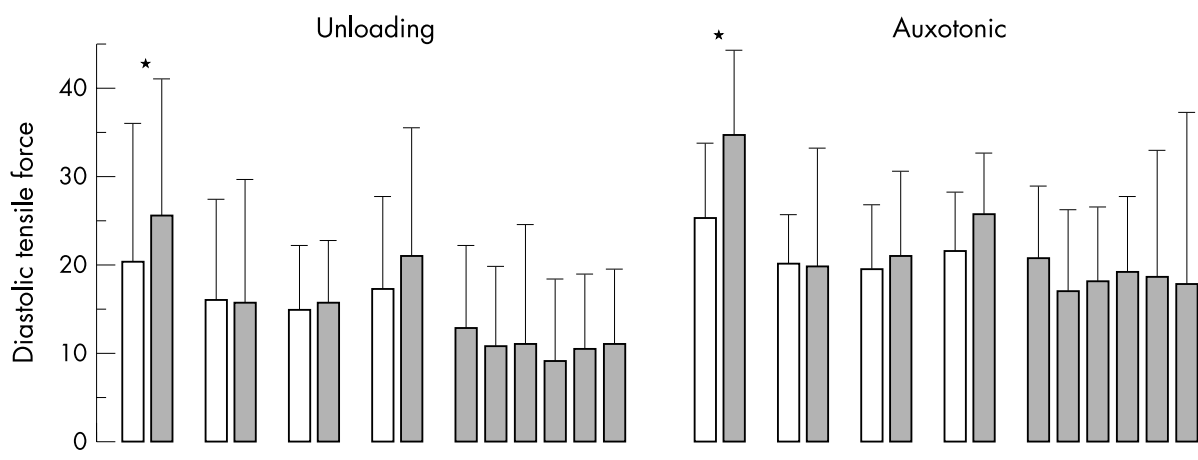

Figure 4 Response of the diastolic (upper), systolic (middle), and developed (lower) tensile force of the unloading (left) and auxotonic (right) type (1) during inflation of the balloon in the aortic root, the addition of (2) $15 \mu \mathrm{g} / \mathrm{min}$ dobutamine (Dobu), (3) $200 \mu \mathrm{g}$ thiopental, and (4) 1.5 (0.7) I of saline given intravenously within 15 minutes, and (5) fast withdrawal of the first 2 I of blood from the left atrium. Notice the significant changes in the averaged systolic auxotonic signal during inotropic interventions and the almost missing changes in the systolic auxotonic signal on withdrawal of volume. The differences in both signals are most significant in developed force.
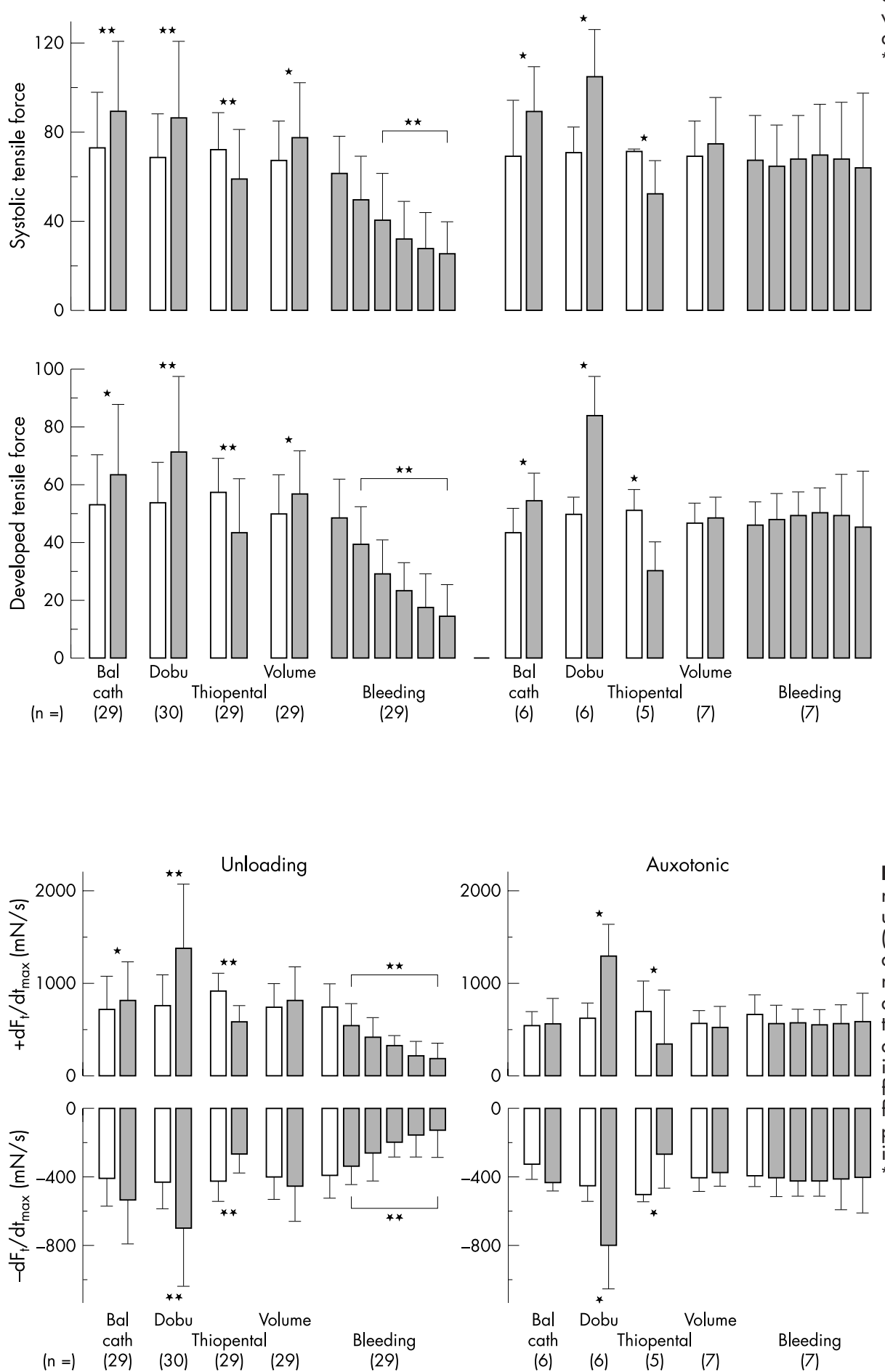

Figure 5 Changes in the velocities in rise (upper) and drop (lower) of the unloading type (left) and auxotonic type (right) of tensile force (1) during an acute increase in left ventricular oufflow resistance, addition of (2) $15 \mu \mathrm{g} / \mathrm{min}$ dobutamine (Dobu) and (3) $200 \mu \mathrm{g}$ thiopental, both given intravenously, and (4) 1.5 (0.7) I saline given intravenously, and (5) withdrawal of the first 2 I of blood within 10 (23) minutes

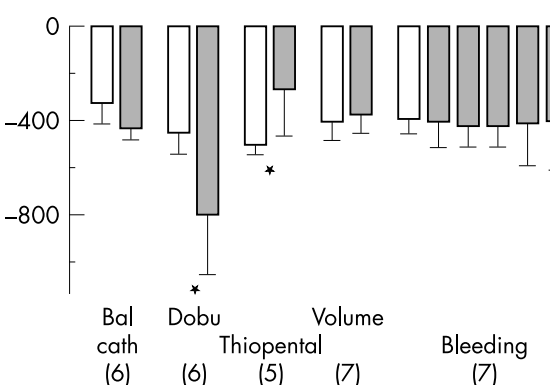

from the left atrium. Note the particularly significant response to inotropic interventions. ${ }^{*} \mathrm{p}<0.05$; ${ }^{* *} p<0.01$. 
A fluid filled catheter was inserted into the left ventricle through the apex to measure left ventricular pressure. We assessed ventricular diameter by implanting ultrasound microcrystals into the epicardium of the superior and inferior equatorial walls. Resistance to outflow from the left ventricle was produced by a balloon catheter inserted into the right femoral artery and advanced into the ascending aorta. Volume and drugs were delivered rapidly through a $5 \mathrm{~mm}$ catheter implanted into the left atrial appendage. The same catheter was used ultimately for bleeding the animals.

All procedures for animal care and experimentation followed the guidelines of the American Physiological Society and the German Law of Animal Protection. The protocol was approved by the University of Munster Institutional Animal Care and Use Committee and adhered to the guidelines for the use of laboratory animals of the National Institutes of Health and Prevention (Der Regierungspräsident Münster, Genehmigungsbescheid 31/ 93, 16.06.1993, and 13.12.1994).

\section{Manoeuvres}

It was our goal to compare the forces generated by the populations of myocardial fibres parallel to the surface, as opposed to those arrayed in oblique transmural fashion, submitting both populations to the variable haemodynamic loads to which the left ventricle is exposed routinely or in extremis.

The balloon in the ascending aorta was inflated for up to 30 heart beats, then deflated. After a 10 minute period of recovery, dobutamine was administered intravenously at a dose of $15 \mathrm{mg} / \mathrm{min}$ over the next 10 minutes, followed by a 20 minute period of recovery. A bolus of thiopental sodium, at $5 \mathrm{mg} / \mathrm{kg}$, was given intravenously to induce transient negative inotropism, followed by a 20 minute period of recovery. Next, $1.5(0.7) \mathrm{l}$ of saline was given within five minutes through the left atrial catheter to establish a left ventricular end diastolic pressure of 18 (9) mm Hg. Finally, cardiodynamic variables were measured during fast exsanguinations, achieved within 19 (7) minutes through the left atrial catheter. With onset of ventricular fibrillation, the hearts were excised and instantly fixed by $10 \%$ formaldehyde flushed repeatedly through the coronary arteries. For the next 72 hours, the hearts were kept submerged in formaldehyde. The sites of implantation of the needle probes were harvested in transmural blocks, with the probes remaining in place during the period of fixation. Histological serial sections were made strictly perpendicular to the long axis of the probe (fig 3).

\section{Definitions}

Our study has shown aspects of ventricular mechanics that are, to some extent, divorced from conventional wisdom. We believe it would be helpful, therefore, to define precisely the way in which our terms are used in the subsequent descriptions.

- Dualism is the putative contractile behaviour of the ventricular myocardium that sustains both a constrictive and a dilatory effect relative to the cavity. It may serve to preserve ventricular shape.

- Mutual stress is the putative contractile impact of all components of the interwoven myocardial wall on each other. This impact is not necessarily contributing to ventricular emptying but may serve to put the wall itself under stress from all directions, thus rendering it more rigid and hence stabilising the ventricular shape. As an analogy, variation of mutual stress in the three orientations of skeletal muscles is known to be the basic mechanism of movement or stiffening of the tongue.
- Tensile force $\left(\mathrm{F}_{\mathrm{T}}\right)$ is the value measured, and calibrated in milli-Newtons, with the needle probes. It corresponds to the force that acts laterally on the inner flexible bar of the probe during contraction of the myocardial fibres spread around the lateral window, engendering tension in the direction of their long axis (fig 1).

- The unloading type of $\mathrm{F}_{\mathrm{T}}$ is the force signal that drops notably during the period of regional fibre shortening (fig 2).

- The auxotonic type of $\mathrm{F}_{\mathrm{T}}$ is the force signal that increases notably during the period of regional fibre shortening (fig 2).

- The period of fibre shortening was timed between the two bends that are observed in the systolic curves generated by both types of $\mathrm{F}_{\mathrm{T}}$ (fig 2). This assumption was validated by making control measurements with microsonometry close to the needle force probes.

\section{Statistical analysis}

All values are expressed as mean (SD). For comparison of paired data we used the Wilcoxon test, and the MannWhitney $U$ test for unpaired data. A probability value of $\mathrm{p}<0.05$ was considered significant. The histogram in fig 6A was generated with the SPSS software package (SPSS Inc, Chicago, Illinois, USA).

\section{RESULTS}

Two distinct types and an indifferent type of $F_{T}$ signals At almost seven tenths of the sites of implantation the needle probes, when inserted, instantly and persistently sensed an unloading type of signal, which dropped while the fibres shortened (fig 2). At about a sixth of sites that were most frequently located near the base or in the apical quarter of the ventricle, however, the probes sensed the auxotonic type of signal, which increased during the period of regional fibre shortening. In the remaining cases, the signal was indifferent, with a slightly domed or dented plateau during fibre shortening. As a feature of both types of development of force, the curves generated had two bends during systole. The first coincided with the onset of focal fibre shortening and the second one we interpret as heralding the end of fibre shortening. With the unloading type of force, the highest level generated coincides with the first bend. In the auxotonic type, in contrast, the force generated reaches its maximum at the second bend. Both points are landmarks for the determination of systolic intervals. As fig $6 \mathrm{~B}$ shows, the time that elapses in any individual heart between the $\mathrm{R}$ wave and the two bends does not necessarily coincide in the two types of force, although the differences are not significant.

\section{Occlusion of the ascending aorta}

Acute augmentation in the resistance to ventricular outflow causes an increase in left ventricular diameter. This is paralleled by an increase in left ventricular systolic and diastolic pressure, and in the velocities of early systolic rise and the drop during relaxation in ventricular pressure (table 1). Acute augmentation in the resistance to ventricular outflow mediates an increase in the unloading type of $\mathrm{F}_{\mathrm{T}}$ (fig 4) and in the velocities of rise and fall (fig 5). The change in the fall is significant. The auxotonic signals rise during systole and diastole (fig 4). The velocities of rise and fall in force, however, do not change significantly (fig 5).

\section{Volume load}

An acute increase in left ventricular filling augments significantly the systolic peak and end diastolic filling pressures, as well as the velocities of early systolic rise and fall during relaxation in left ventricular pressure (table 1). 

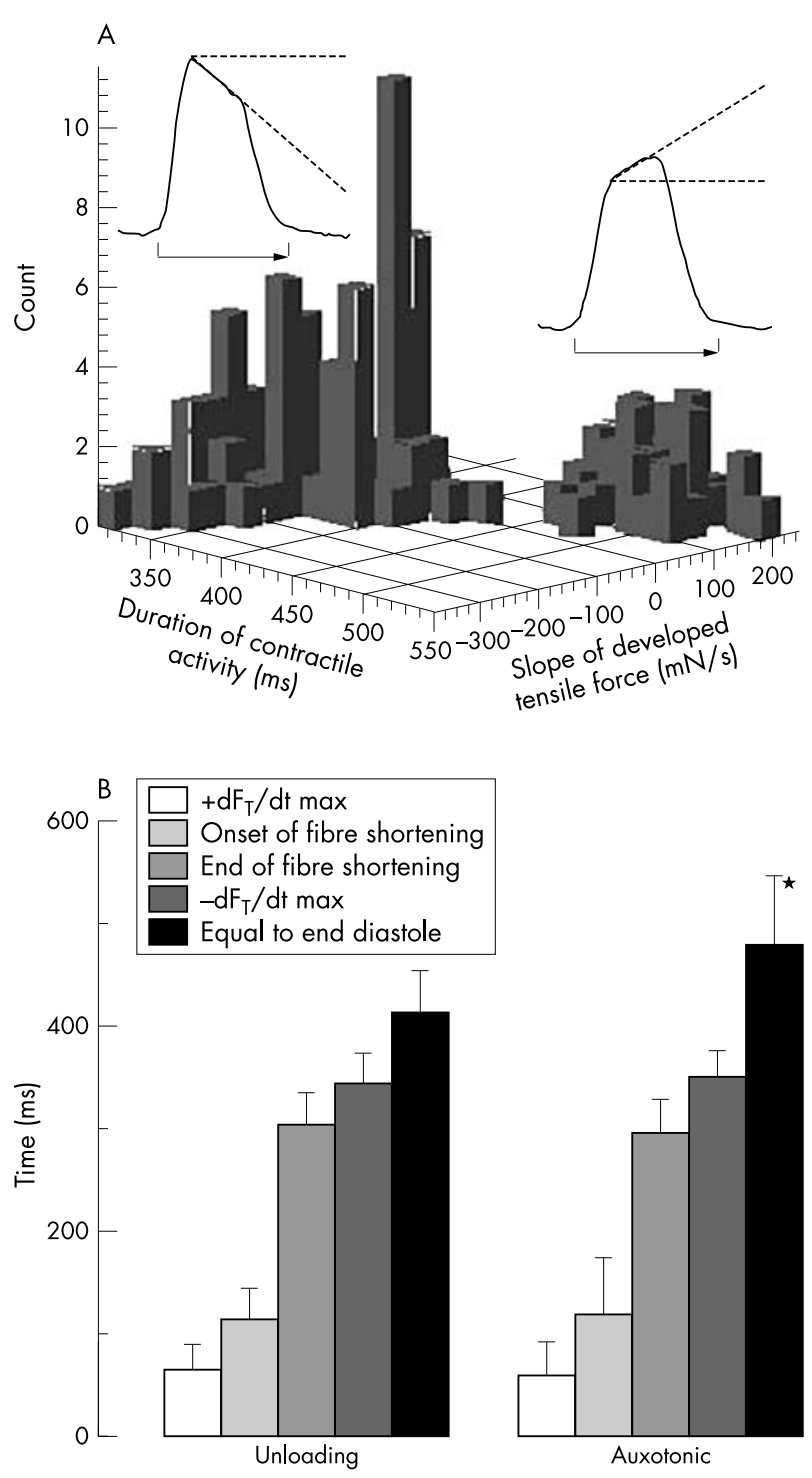

Figure 6 (A) Histogram of the duration of contractile activity versus slope in tensile force. The duration of activity is the time from the onset at the peak of the $R$ wave until the corresponding level of tensile force has been reached at the end of systole. The duration of contractile activity was short in the unloading type of force (left) and long in the auxotonic type (right). The number of curves counted in the graph is the number of the measuring sites in all animals times four, since at each site measurements were made during two heart cycles, each at two extreme states of ventricular filling. (B) Systolic intervals from the peak of the $R$ wave to the maximum velocity of rise in tensile force, the onset of fibre shortening, the end of fibre shortening, the maximum velocity of the drop in force during relaxation, and the end of activity when force reaches the same level as during the peak of the $\mathrm{R}$ wave. Note that only the duration of the last systolic interval in the auxotonic signal is significantly different from that in the unloading type of signal. ${ }^{*} \mathrm{p}<0.01$.

The unloading type of force signal increases during systole and diastole (fig 4), the latter less dramatically than the rise in left ventricular pressure. So do the velocities of rise and fall in force (fig 5). The changes in auxotonic force (fig 4) and in the velocities in rise and fall of auxotonic force (fig 5) are not significantly changed with administration of volume.

\section{Positive inotropic stimulation}

Dobutamine administered intravenously at $0.05 \mu \mathrm{g} / \mathrm{kg} / \mathrm{min}$ induces a significant increase in left ventricular systolic pressure but no appreciable change in end diastolic pressure. In contrast, the velocities of rise and fall of pressure increase significantly (table 1). Diastolic $\mathrm{F}_{\mathrm{T}}$ of the unloading type do not increase (fig 4) but there is a highly significant increase in systolic $\mathrm{F}_{\mathrm{T}}$ and in the velocities in rise and fall in those forces (fig 5). Auxotonic force increases significantly during systole but does not change appreciably during diastole (fig 4). The velocities of rise and fall in auxotonic force increase significantly (fig 5).

\section{Left ventricular unloading by exsanguination}

With the onset of rapid withdrawal of blood from the left atrium, 3.5-5 l within 19 (7) minutes, left ventricular pressure commences to drop exponentially and significantly during both systole and diastole. So do the velocities of early systolic rise and fall in pressure during relaxation (table 1). The pattern of the unloading type of $\mathrm{F}_{\mathrm{T}}$ follows the exponential drop in the left ventricular pressure (fig 4), as do the velocities of rise and fall of this tension (fig 5). The auxotonic force, however, does not change significantly, during neither systole nor diastole. With the onset of bleeding, the auxotonic force increases slightly in the mean (fig 4). It increases more substantially in some areas (fig 2) than in others. The velocities of rise and fall in auxotonic force do not change significantly (fig 5 ).

\section{Duration of contractile activity}

The time that elapsed from the peak of the $\mathrm{R}$ wave until the end of contractile activity when the signal of $\mathrm{F}_{\mathrm{T}}$ had dropped to the end diastolic level was closely related to the slope of the measured forces while the fibres were shortening (fig 6). The unloading force, which falls while the fibres shorten, ended significantly earlier, at the earliest $328 \mathrm{~ms}$ and at the latest $467 \mathrm{~ms}$ with a mean of 412 (37) ms, than the auxotonic type of force. The latter value was seen at the earliest $402 \mathrm{~ms}$ and at the latest $614 \mathrm{~ms}$, and had a mean of 478 (67) ms. The other systolic intervals did not differ significantly between the two types of signals (fig 6).

\section{Histological findings}

Histological analysis of the sites of implantation of the needle probes in the base and apex showed a prevalent oblique transmural inclination of the network in the direct vicinity of the bed of the needle in those areas where an auxotonic signal had been persistently measured (fig 3). The angle of deviation with respect to the epicardial and endocardial surfaces and the number of oblique fibres varied widely between the specimens. In contrast, in most areas in the midportion of the left ventricle the network around needles that had sensed an unloading signal was arranged parallel to the epicardial surface (fig 3). Within the overall series of our histological preparations, we found a more or less continuous distribution of angles of inclination. In keeping with these morphological findings, we found a continuum of transient types of force signals between the unloading and the auxotonic types. We rarely found erythrocytes, oedema, or an accumulation of plasma proteins directly adjacent to the site of implantation of the probes, indicating that there had been minimal trauma during the introduction of the needles. Some of the innermost myocardial fibres, however, were lacerated. The disruption of branching of the fibres was less pronounced in the area of the window than elsewhere in relation to the probe.

\section{DISCUSSION}

Although there is a wealth of anatomical work devoted to the orientation of the myocardial fibres within the ventricular wall, ${ }^{6-811-15}$ little attention has been paid to the notable regional inhomogeneities in fibre orientation. Nor has the question been addressed as to whether the morphological variations cause disparities in regional function. ${ }^{9}$ The myocardium is arranged with each of the fibres attached to 
Table 1 Alterations in left ventricular pressure and derivatives and the outer left ventricular diastolic diameter during acute increase in left ventricular outflow resistance, administration of $15 \mu \mathrm{g}$ dobutamine, $200 \mu \mathrm{g}$ thiopental given intravenously, and $2000 \mathrm{ml}$ saline given intravenously, and withdrawal of 2 I of blood within 10 (3) minutes from the left atrium

\begin{tabular}{|c|c|c|c|c|c|}
\hline & LVEDP $(\mathrm{mm} \mathrm{Hg})$ & LVP $\max (\mathrm{mm} \mathrm{Hg})$ & $+\mathrm{dP} / \mathrm{dt}_{\max }(\mathrm{mm} \mathrm{Hg} / \mathrm{s})$ & $-\mathrm{dP} / \mathrm{dt}_{\max }(\mathrm{mm} \mathrm{Hg} / \mathrm{s})$ & LVEEDD(\% v control) \\
\hline Aortic occlusion & $8(3) \rightarrow 14(6)^{\star *}$ & $111(18) \rightarrow 150(24)^{\star *}$ & $1753(399) \rightarrow 1769(276)$ & $1420(380) \rightarrow 1796(322)^{* *}$ & $2.8(3.1)^{\star *}$ \\
\hline Dobutamine & $13(5) \rightarrow 13(9)$ & $119(21) \rightarrow 166(33)^{* *}$ & $1710(407) \rightarrow 3155(1016)^{* *}$ & $1517(324) \rightarrow 2226(732)^{* *}$ & $-1.9(2.7)$ \\
\hline Thiopental & $11(5) \rightarrow 9(2)$ & $128(34) \rightarrow 86(36)^{*}$ & $1973(572) \rightarrow 1207(609)^{*}$ & $1640(196) \rightarrow 822(156)^{*}$ & $0.7(1.9)$ \\
\hline Volume load & $9(3) \rightarrow 118(8)^{\star *}$ & $102(15) \rightarrow 120(21)^{\star *}$ & $1450(351) \rightarrow 1735(402)^{*}$ & $1225(345) \rightarrow 1413(378)^{*}$ & $4.7(7.2)^{*}$ \\
\hline \multicolumn{6}{|l|}{ Bleeding } \\
\hline Start & $10(4)$ & $120(23)$ & $1810(440)$ & $1459(437)$ & $(100 \%=51 \mathrm{~mm})$ \\
\hline $20 \%$ & $6(3)^{* *}$ & $106(25)^{* *}$ & $1492(423)^{* *}$ & $1198(411)^{* *}$ & $-3.9(4.5)^{\star \star}$ \\
\hline $40 \%$ & $4(2)^{* *}$ & $86(19)^{* *}$ & $1257(418)^{* *}$ & $887(286)^{* \star}$ & $-6.4(3.5)^{* *}$ \\
\hline $60 \%$ & $3(2)^{* *}$ & $73(24)^{* *}$ & $1080(343)^{* *}$ & $790(343)^{* *}$ & $-10.9(5.2)^{\star *}$ \\
\hline $80 \%$ & $3(3)^{* *}$ & $56(24)^{* *}$ & $796(391)^{* *}$ & $592(267)^{* *}$ & $-13.5(6.3)^{* *}$ \\
\hline End & $2(2)^{* *}$ & $47(25)^{* *}$ & $735(448)^{* *}$ & $473(275)^{* *}$ & $-15.3(7.2)^{* *}$ \\
\hline
\end{tabular}

Values are mean (SD), $n=7$. Indication of $20 \%$ to $80 \%$ means that $20-80 \%$ of $2 \mathrm{I}$ blood had been withdrawn. LVEDP, left ventricular end diastolic pressure; LVEEDD, left ventricular epicardial end diastolic diameter; LVP, left ventricular pressure; ${ }^{*} \mathrm{p}<0.05$; ${ }^{* *} \mathrm{p}<0.01$.

its neighbours. ${ }^{11-14}$ By dividing spatially, unlike railways, each myocyte is a constituent of a space lattice. The number of branches arising from any individual myocyte and the direction of the offshoots vary greatly within the myocardial mass. ${ }^{13}$ The overall effect is to produce a dense myocardial network that gives rise to an intricate pattern of forces, acting not only in the planes of the ventricular surfaces but also in oblique and transmural directions. It is the specific alignment of the fibres within this complex mural meshwork that determines the characteristics of the $\mathrm{F}_{\mathrm{T}}$ measured in the wall. Recently propounded concepts of myocardial structure, arguing that the ventricular musculature is arranged in the form of a unique myocardial band, ${ }^{16}$ are incompatible with the intricate pattern of functional differentiation shown by our measurements. Our findings, in contrast, are entirely compatible with the concept of the heart being a modified blood vessel, with each myocardial fibre attached to its neighbour to form a heterogeneous meshwork. ${ }^{11}$ Our present histological findings have also validated our hypothesis that the disparate working conditions for the tangential and oblique transmural components of the myocardium would generate two types of force signal. We have shown that each type of signal exhibits transients over the heart cycle that comply with the conformational changes of the ventricle and its global loading conditions.

\section{Probe to fibre coupling}

Unlike the coupling modalities in skeletal muscle fibres, which do not branch, ${ }^{3}$ in the myocardium measuring contractile forces with needle force probes profits from the structured meshwork arrangement. The probe spreads the tissue, the shape of the probe determining the geometry of deviation of the individual fibres. The branching myocardial fibres themselves grip the probe tightly like a sphincter, so that those surrounding the probe conform precisely to its shape (fig 1, fig 3). When the probe is turned along its long axis, therefore, the drop in the amplitude recorded from the myocardium is less pronounced than that recorded in skeletal muscle, but it is still measurable. ${ }^{3}$ When the probe is implanted and the shield has been pulled back from the lateral window, the fibres that slip into the indentation at the window exert a force that slightly deviates the inner flexible bar. In figures, a measured load of $100 \mathrm{mN}$ corresponds to a deviation of $50 \mu \mathrm{m}$. The fibres that can slip into the lateral window (fig 1) are those that cross the probe at approximately a right angle relative to its long axis. Reliable coupling, and hence the maximum signal, are obtained when the fibres in the indentation are aligned parallel to the plane of the window. Some basic knowledge of the alignment of the myocardial fibres, therefore, is a prerequisite for adequate implantation of the probes. In fact, by following the alignment of the fibres, shown by Streeter and colleagues ${ }^{4}$ to rotate on a radial axis from the epicardium to the endocardium, it is possible to couple the probe confidently to any area of interest. In detail, using a quasitangential implantation (fig 3), with the window directed towards the epicardium, we sample the predominant number of fibres aligned tangentially to the epicardium. When oblique transmural pathways prevail in an area of interest (fig 3), the plane of the window needs to be adjusted to the obliquity of the fibres. This is achieved by rotating the probe minimally along its long axis until the maximum signal is recorded. In all instances, we inserted the probe at right angles to the alignment of the fibres to be measured. The resulting type of signal, depending on the prevalence of a strictly tangential or oblique transmural alignment of the local array, was found to be random in any given area. The auxotonic type, none the less, was found more often in the deep layers of the base and in the apex than in other areas.

It may be thought that our findings could have been distorted by trauma induced by insertion of the needle into the myocardium. ${ }^{1}$ Stein and colleagues, ${ }^{17}$ however, showed that catheter tipped manometers can be inserted into the myocardium in a relatively atraumatic fashion. Like them, we did not encounter bleeding, oedema, or sequestration of plasma proteins around the site of insertion of our probes. Furthermore, spreading the myocardial fibres with a needle produces no more than reversible and minor trauma, as shown in the daily routine of the cardiac surgeon, who punctures the ventricular cavity to measure pressure without ensuing bleeding. Thus, we do not believe that our measurements have been distorted by the trauma produced by insertion of the probes.

\section{Potential disparities in the freedom of the myocytes to shorten}

As already emphasised, most existing analyses relating to cardiodynamic data are based on the assumption that the myocardial fibres are aligned in planes that are tangential to the endocardial and epicardial surfaces of the chambers. ${ }^{2} 1314$ This has been justified on the observation that there is a prevalence of fibres running parallel to the surface. ${ }^{68^{11-15}}$ It is presumed that, because of this dominant orientation of the fibres, the forces generated by myocytic contraction are, in essence, tangential. It was on this assumption that Frank ${ }^{2}$ established his cardiodynamic concept, arguing that all resulting forces serve to contract the ventricular walls against the intracavitary pressure. Several investigators, none the less, have shown the existence of oblique transmural fibres interspersed with the fibres parallel 
to the surface. ${ }^{6-8} 1112$ To date, however, their influence has been considered only secondary with regard to the function of the ventricular pump. ${ }^{18}$ Considering the substantial number of fibres oriented in directions that deviate appreciably from the plane that is tangential to the surface, ${ }^{68}$ it seems to us inappropriate to dismiss their effects in the analysis of ventricular function. It is precisely this population of fibres that we have addressed in this study. We submit that our results testify to their importance.

In this respect, it is significant that, almost a century ago, Keith ${ }^{19}$ suggested the musculature of the iris as a model for systolic ventricular function, assuming that all fascicles of the myocardium ultimately terminated in the internal spiral coat, just as every segment of the diaphragm of the iris takes part in forming the margin of its pupil. He might have opined that those fibres intruding as spiralling contractile pathways were freely movable, just like the rope in a pulley block. Then their oblique intruding orientation probably would not be associated with an increase in force during systolic wall thickening. This concept, however, ignores the fact that the dense netting of the intruding fibres with the tangential fibres confines their freedom of motion. Because of those constraints, the population of intruding fibres in the myocardial mass is hindered in its capacity to shorten while mural thickness increases during systole.

It may be argued that this model of the iris is also compatible with the concept espoused by Torrent-Guasp, ${ }^{16}$ namely that a continuous muscular band wraps round the ventricular cavities in three loops. The basic assumption underscoring this concept, ${ }^{16}$ however, is fallacious. As with the concept of Keith, ${ }^{19}$ the putative unique muscular band is supposed to be freely movable on itself, like the rope in a pulley block. In fact, in the case of Torrent-Guasp, ${ }^{16}$ the apparent freedom is no more than the artefact introduced by preparation of the specimens, during which destructive process the myriad linkages between the layers are disrupted. Our observation showing that mural thickening is associated with an increase in force in the population of obliquely intruding fibres points to the inadequacy of any theory based on the concept of a freely movable spiralling muscle likened to a pulley block.

The loading conditions of fibres oriented parallel and oblique to the surfaces, respectively, are not necessarily uniform. Our data indicate that conformational changes of the left ventricle, which are associated with major changes in the thickness of its wall, mediate a measurable redistribution of $\mathrm{F}_{\mathrm{T}}$. Concomitant with the increase in mural thickness seen with withdrawal of blood volume, there is an increase in the resistance to shortening of the oblique fibres. Overall, we regularly measure either no decrease (fig 4) or a major increase (fig 2) in the developed auxotonic force. Since diastolic force remains essentially unchanged, we assume that, while mural thickness increases, the intruding fibres are erected in a radial direction without being further elongated. In contrast, during systole while the heart shrinks, the oblique fibres are particularly hindered in their shortening. The same effect is encountered during systole in the normally filled left ventricle. Here also, the oblique fibres cannot shorten to the same extent as the tangential fibres. While the wall thickens, the oblique transmural fibres are progressively hindered in shortening, resulting in an auxotonic type of force signal. In contrast, fibres that are parallel to the surfaces shorten as the ventricle shrinks during systole, and similarly during exsanguination. Accordingly, their actively developed force decreases. In other words, the two extremes of prevailing orientations of fibres are acting under very disparate working conditions.

The essence of our argument is that all myocardial components are acting with the same basic contractile properties. We relate the remarkably bimodal observed behaviour of contraction to the regional disparities in the cyclical realignment of the myocardial fibres. Recent investigations in wall motion based on magnetic resonance tomography ${ }^{20}$ have found distinctly individual patterns in focal mural thickening, both in amount and in timing, which points to gradients in focal constraints. These findings are entirely compatible with observed structural disparities such as the major variations in the density of the spatial myocardial netting. ${ }^{6-8} 111215$

\section{Freedom to shorten and the duration of contractile activity}

According to Jewell and Wilkie ${ }^{21}$ and to Brutsaert and colleagues, ${ }^{22} 23$ the duration of the contractile cycle is essentially determined by the freedom of the fibres to shorten. The duration of peak mechanical activity is shortest after isotonic contraction and longest after isometric contractions. During ventricular ejection, the myocytes within the ventricular wall that are aligned parallel to the epicardial or endocardial surface planes are progressively unloaded. They are consequently free to shorten. Our results, therefore, confirm that these fibres exhibit the shortest period of mechanical activity. The oblique transmural fibres, in contrast, exerting auxotonic contractions, persist the longest in the state of mechanical activity because of their confined freedom to shorten. In detail, fig 6 shows the duration of mechanical activity is related to the steepness of the rise or drop of the force during ventricular ejection. The steeper the rise in auxotonic force, the longer the mechanical activity persists. In contrast, the steeper the drop in the unloading type of force, the shorter the persistence of mechanical activity. In accordance with Jewell and Wilkie, ${ }^{21}$ therefore, we consider it justifiable to infer that the various types of force signals are related to variable focal loading conditions, rather than assuming that essential differences in intrinsic contractile properties prevail between adjacent myocardial compartments.

We have focused on the evaluation of two of the most characteristic types of tracings, namely the unloading and the auxotonic type of $\mathrm{F}_{\mathrm{T}}$. We have also measured routinely, none the less, a great variety of transient force signals in mammals. ${ }^{3}{ }^{10}$ This continuous modulation in function, from the most frequent unloading type of force signal to the less frequent notably auxotonic signal, is in keeping with the continuous redistribution of angles of inclination of the myocardial net from the prevailing tangential population to the oblique and transmural.

Our study, in compliance with established knowledge on basic mechanisms in myocardial contraction, shows that such auxotonic contraction is associated with a prolongation of contractile activity. It is modulated to some extent by ventricular size and shape, but most sensitively by inotropic interventions. We conclude that the forces simultaneously engendered by fibres aligned in continuously varying directions within the ventricular wall may serve to put mutual stress on each other to preserve the proper shape of the ventricle. Negative inotropic medication induced a highly significant drop in the auxotonic type of $\mathrm{F}_{\mathrm{T}}$. This fall was appreciably less dramatic in the fibres parallel to the surface, these fibres generating the unloading type of tension. Thus, slackening one rein of the dualistic system of control, specifically by attenuating the auxotonic component of the force with negative inotropic medication, tempers the mutual interplay of the forces. This mechanism potentially attenuates the undesired side effect of hypertrophy on compliance. 


\section{ACKNOWLEDGEMENTS}

Supported by: Deutsche Forschungsgemeinschaft, Karl und Lore Klein Stiftung, Ernst und Berta Grimmke Stiftung, and the British Heart Foundation.

\section{Authors' affiliations}

P P Lunkenheimer, K Redmann, J Florek, Klinik und Poliklinik für Thorax-, Herz- und Gefäßchirurgie, University Münster, Munster, Germany

U Fassnacht, Institut für Anatomie, University Münster, Munster,

Germany

C W Cryer, F Wübbeling, Institut für Numerische Mathematik, University Münster, Munster, Germany

P Niederer, Institute of Biomedical Engineering, ETH und Universität Zürich, Zurich, Switzerland

R H Anderson, Cardiac Unit, Institute of Child Health, University College London, London, UK

\section{REFERENCES}

1 Huisman RM. Forces in the wall of the left ventricle. Academisch Proefschrift. Amsterdam: Druck erla, 1977.

2 Frank O. Isometrie und Isotonie des Herzmuskels. Z Biol 1901:41:14-34.

3 Lunkenheimer PP, Lunkenheimer A, Stroh N, et al. Vergleich klassischer und neuer methodischer Zugänge zum intramyokardialen Kraftiverteilungsmuster. Zentralb/ Veterinarmed 1982;29:557-601.

4 Feigl EO, Simon GA, Fry DF. Auxotonic and isometric cardiac force transducers. J Appl Physiol 1967;23:597-600.

5 Walton RP, Brodie OJ. The effect of drugs on the contractile force of a section of the right ventricle under conditions of an intact circulation. J Pharmacol Exp Ther 1947;90:26-41.

6 Cryer CW, Navidi-Kasmai H, Lunkenheimer PP, et al. Computation of the alignment of myocardial contractile pathways using a magnetic tablet and an optical method. Technol Health Care 1997;5:79-93.

7 Jouk PS, Usson Y, Michalowicz B, et al. Three-dimensional cartography of the pattern of the myofibres in the second trimester fetal human heart. Anat Embryol 2000;202:103-18.
8 Lunkenheimer PP, Redmann K, Dietl KH, et al. The heart's fibre alignment assessed by comparing two digitizing systems: methodological investigation into the inclination angle towards wall thickness. Technol Health Care 1997:5:65-77.

9 Redmann K, Lunkenheimer PP, Scheld HH, et al. Inhomogeneities in wall stress measured by microergometry in the heart muscle in situ. Technol Health Care 1997;5: 123-34.

10 Lunkenheimer PP, Lunkenheimer A, Whimster WF, et al. Local myocardial and global ventricular function compared during positive inotropic medication. Basic Res Cardiol 1986:81(suppl):59-71.

11 Anderson RH, Becker AF. The orientation of fibres within the ventricular mass. In: Cardiac anatomy. London: Churchill Livingstone, 1980:5.14-5.26.

12 Greenbaum RA, Ho SY, Gibson DG, et al. Left ventricular fibre architecture in man. Br Heart J 1981;45:550-8.

13 LeGrice IJ, Smaill BH, Chai LZ, et al. Laminar structure of the heart: ventricular myocyte arrangement and connective tissue architecture in the dog. Am J Physiol 1995;269:H571-82.

14 Streeter DD Jr. Gross morphology and fiber geometry of the heart. In: Berne RM, Sperelakis N, Geiger SR, eds. The cardiovascular system, 1 Section 2, Handbook of physiology. Bethesda, Maryland: American Physiological Society, 1979:61-112,

15 Sanchez-Quintana D, Garcia-Martinez V, Hurle JM. Myocardial fiber architecture in the human heart. Acta Anatom 1990;138:352-8.

16 Torrent-Guasp F, Buckberg GD, Clemente C, et al. The structure and function of the helical heart and its buttress wrapping. I. The normal macroscopic structure of the heart. Semin Thorac Cardiovasc Surg 2001;13:301-319.

17 Stein PE, Marzilli M, Sabbah HN, et al. Systolic and diastolic pressure gradients within the left ventricular wall. Am J Physiol 1980;238:H625-30.

18 Bovendeerd PHM, Huyghe JM, Arts T, et al. Influence of endocardialepicardial crossover of muscle-fibers on left ventricular wall mechanics. J Biomech 1994; 27:941-51.

19 Keith A. The Functional anatomy of the heart. BMJ 1918;i:316-63.

20 Tseng WY, Wedeen VJ, Reese TG, et al. Diffusion tensor MRI of myocardial fibers and sheets: Correspondence with visible cut-face texture. J Magn Reson Imaging 2003;17:31-42.

21 Jewell BR, Wilkie ER. The mechanical properties of relaxing muscle. J Physiol (Lond) 1960;152:30-47.

22 Brutsaert DL, De Clerck NM, Goethals MA, et al. Relaxation of ventricular cardiac muscle. J Physiol 1978;283:469-80.

23 Brutsaert DL, Housmans PR, Goethals MA. Dual control of relaxation. Its role in the ventricular function in the mammalian heart. Circ Res 1980;47:637-52.

\section{IMAGES IN CARDIOLOGY}

\section{Catheter induced multiple spasms in the right iliac artery during a percutaneous coronary intervention}

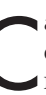

atheter induced vasospasm of small calibre arteries, such as coronary, radial, or brachial arteries, is frequently observed during cardiac catheterisation, but the phenomenon in large calibre arteries has rarely been reported. We report a case in which the right iliac artery had multiple spasms during percutaneous coronary intervention (PCI) via the right femoral approach in a patient who presented with acute myocardial infarction. He was found to have acute ST segment elevation myocardial infarction. A coronary angiogram revealed a total thrombotic occlusion in the mid left anterior descending coronary artery (LAD). Primary PCI for the LAD lesion was performed with the aid of abciximab. An abdominal aortogram after the PCI revealed multiple narrowings along the right external iliac artery, causing the artery to appear like a corkscrew (left panel). However, these narrowings completely resolved without any medication or intervention (right panel).

Detection of this pseudo-lesion helped to avoid unnecessary intervention and complications.
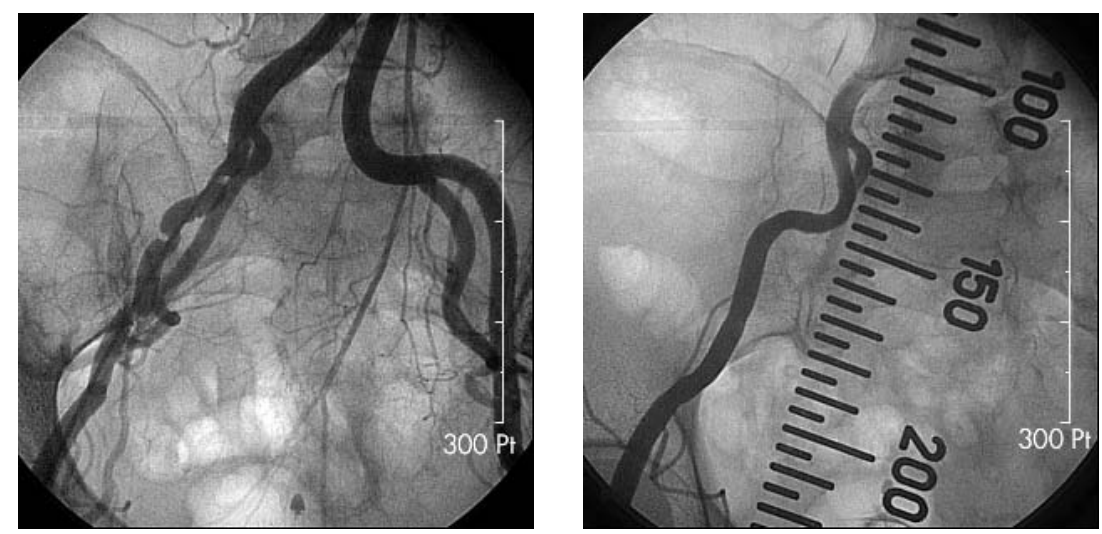